\title{
Reversibly Light-Switchable Wettability of Hybrid Organic/Inorganic Surfaces With Dual Micro-/ Nanoscale Roughness
}

\author{
By Gianvito Caputo, Barbara Cortese, Concetta Nobile, Marco Salerno, \\ Roberto Cingolani, Giuseppe Gigli, Pantaleo Davide Cozzoli, and \\ Athanassia Athanassiou*
}

\begin{abstract}
Here, an approach to realize "smart" solid substrates that can convert their wetting behavior between extreme states under selective light irradiation conditions is described. Hybrid organic/inorganic surfaces are engineered by exploiting photolithographically tailored SU-8 polymer patterns as templates for accommodating closely packed arrays of colloidal anatase $\mathrm{TiO}_{2}$ nanorods, which are able to respond to UV light by reversibly changing their surface chemistry. The $\mathrm{TiO}_{2}$-covered SU-8 substrates are characterized by a dual micro-/nanoscale roughness, arising from the overlapping of surfactantcapped inorganic nanorods onto micrometer-sized polymer pillars. Such combined architectural and chemical surface design enables the achievement of UV-driven reversible transitions from a highly hydrophobic to a highly hydrophilic condition, with excursions in water contact angle values larger than $100^{\circ}$. The influence of the geometric and compositional parameters of the hybrid surfaces on their wettability behavior is examined and discussed within the frame of the available theoretical models.
\end{abstract}

\section{Introduction}

Over the past decade, the control of the wetting properties of solid surfaces has attracted considerable attention due to its implications in technological fields, such as microelectronics, sensing, fabrication of self-cleaning and antifogging materials, manipulation of biomolecules and cells, and microfluidics. ${ }^{[1-50]}$ Since the wetting properties of materials are governed by the joint effects of their inherent chemical nature and surface geometrical arrangement, tailored surfaces that exhibit specific wetting characteristics can be obtained by appropriate compositional and structural engineering. To this purpose, a variety of organic and inorganic materials with textured roughness and distinct chemical features have been engineered by exploiting, on one hand, different fabrication tools - such as chemical-vapor or sol-gel deposition, lithographic patterning, programmed assembly of colloidal nanoparticles - and, on the other hand, refined chemical surface modification techniques - including plasma-assisted or reactive-ion etching and wet-chemical grafting. . $^{[1,3,4,6-29,44]}$

A more challenging direction in this field of research deals with the fabrication of surfaces capable of switching their wettability under suitable external stimulation. One class of strategies involves surface functionalization with particular organic molecules that can exhibit conformational transitions and/or dipole moment variation upon exposure to external stimuli, such as light, electrical potential, heating, solvent, or $\mathrm{pH}$ changes. ${ }^{[13-19]}$ Another approach exploits the ability of some semiconductor
[*] Dr. A. Athanassiou, Prof. R. Cingolani

NNL-National Nanotechnology Laboratory of CNR-INFM

Distretto Tecnologico ISUFI

Via per Arnesano, 73100 Lecce (Italy)

and

IIT-Italian Institute of Technology

Via Morego 30, 16152 Genova (Italy)

E-mail: athanassia.athanassiou@unile.it

G. Caputo, B. Cortese, Dr. C. Nobile, Prof. G. Gigli

NNL-National Nanotechnology Laboratory of CNR-INFM

Distretto Tecnologico ISUFI

Via per Arnesano, 73100 Lecce (Italy)
Dr. M. Salerno

IIT-Italian Institute of Technology

Via Morego 30, 16152 Genova (Italy)

Dr. P. D. Cozzoli

NNL-National Nanotechnology Laboratory of CNR-INFM

Distretto Tecnologico ISUFI

Via per Arnesano, 73100 Lecce (Italy)

and

Scuola Superiore ISUFI

Università del Salento, Distretto Tecnologico ISUFI

Via per Arnesano km 5, 73100 Lecce (Italy) 
oxides, such as $\mathrm{TiO}_{2}, \mathrm{WO}_{3}, \mathrm{ZnO}$, and $\mathrm{V}_{2} \mathrm{O}_{5}$, to increase the degree of surface hydroxylation and, hence, hydrophilicity, under band-gap photoexcitation. Most commonly, a recovery of the initial condition takes place upon storage in the dark under air. ${ }^{[20-32]}$ As compared to stimuli-responsive organic molecules, such light-sensitive transition metal oxides are characterized by a higher structural and photochemical stability and can exhibit inherently larger wettability changes (of the order of $30-70^{\circ}$ ) already in the form of selected singlecrystal surfaces or relatively smooth polycrystalline films.

More recently, significant progress in the manipulation of the light-induced wettability changes of inorganic oxides has been reported. For example, amplification of the photochemically driven response has been demonstrated for wet-chemically prepared nanostructured films that consist of organized arrays of oxide nanorods protruding out of a substrate. ${ }^{[27-30]} \mathrm{In}$ such cases, reversible superhydrophobic to superhydrophilic conversions have been ultimately attained. ${ }^{[27-30]}$ Further developments include the realization of hydrophobic and hydrophilic micron-sized patterns over continuous nanometer-sized roughened oxide films by means of photomask-controlled illumination $^{[33-37]}$ or photocatalytic lithography, ${ }^{[38,39]}$ or by preparation of substrates that exhibit alternating regions of dissimilar oxides upon combination of photolithography and plasmaenhanced chemical vapor deposition. ${ }^{[40]}$ The resulting surfaces exhibit large wettability contrast properties, which can be exploited to guide water condensation and selective deposition of polymer nanoparticles. ${ }^{[33,35,36,40]}$ However, although the ability to directly generate micron-scale patterns made of oxide nanostructure arrays on suitably treated substrates has been advanced remarkably, ${ }^{[10,41-50]}$ it has only occasionally been exploited to enable dynamic wettability behavior. ${ }^{[46]}$

Here, we describe an approach to realize "smart" solid surfaces that can modify their wetting behavior under selective light irradiation conditions. In particular, hybrid organic/ inorganic surfaces were engineered by exploiting photo-lithographically tailored SU-8 polymer patterns as templates for accommodating close-packed thin films of colloidal anatase $\mathrm{TiO}_{2}$ nanorods, which are able to respond to UV light by reversibly changing their surface chemistry. The $\mathrm{SU}-8 / \mathrm{TiO}_{2}$ substrates prepared offer a dual micro-/nanoscale roughness, arising from the superimposition of the surfactant-capped inorganic nanocrystals onto the micrometer-sized polymer pillars. Such suitably combined architectural and chemical surface design enables the achievement of UV-driven transitions from a highly hydrophobic to a highly hydrophilic condition, with excursions in water contact angle (WCA) of more than $100^{\circ}$. A dark storage period of a few weeks allows the native hydrophobicity of the surfaces to be restored. The influence of the structural and chemical parameters of the hybrid surfaces on their wettability behavior is examined and discussed. To the best of our knowledge, this is the first report combining the light-induced and reversible wettability changes of inorganic oxides with micron-scale patterned polymeric systems, which may be promising in various applicative fields, such as microfluidics. ${ }^{[50 b-d]}$

\section{Results and Discussion}

In this work, we have realized smart surfaces exhibiting lightswitchable wettability that can reversibly change from a highly hydrophobic to a highly hydrophilic state. Our strategy, summarized in Scheme 1, aims at achieving surfaces with a 


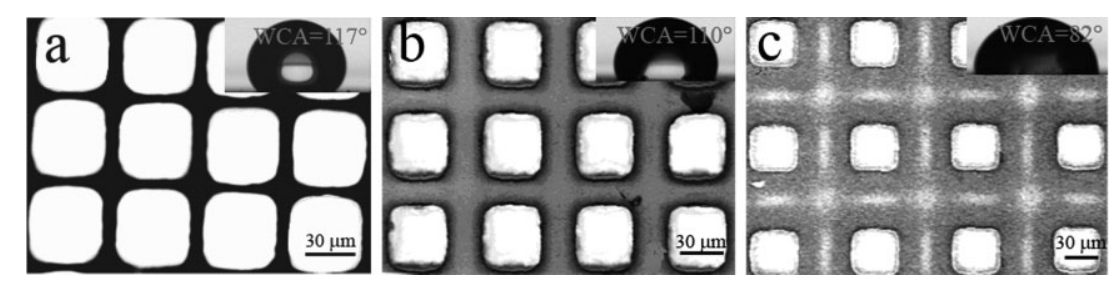

Figure 1. Low-magnification top-view SEM images of pillared SU-8 patterns fabricated onto $\mathrm{SiO}_{2} / \mathrm{Si}$ wafers, each comprising equally sized $42 \times 42 \mu \mathrm{m}$ pillars that are spaced from each other by different distances: a) 14 , b) 28 , and c) $77 \mu \mathrm{m}$. In the corresponding insets, the optical images of a water droplet in contact with each surface are shown.

dual scale roughness arising from the combination of organic micrometer-sized patterns with UV-responsive colloidal inorganic oxide nanocrystals. Specifically, patterns consisting of micron-sized polymeric pillars are fabricated by photolithographic processing of $\mathrm{SU}-8$ on $\mathrm{SiO}_{2} / \mathrm{Si}$ supporting wafers. Patterning of SU-8 results in masters with desirable requirements, such as selected geometric parameters, elevated mechanical toughness, and chemical resistance to most common organic solvents, making them suitable for microfluidic structures. ${ }^{[50 \mathrm{~b}-\mathrm{d}]}$ The micrometric structuring offers a tool to increase the hydrophobicity of the organic surface, compared to the corresponding flat counterpart in a gradual and systematic manner $\left(\mathrm{WCA}_{\mathrm{II}}>\mathrm{WCA}_{\mathrm{I}}\right)$ by proper tailoring of the pillar geometry (Scheme 1, panels I and II). Following this stage, wet-chemically synthesized anatase $\mathrm{TiO}_{2}$ nanorods (NRs), which are fully soluble in non-polar media (e.g., chloroform, toluene and hexane) due to their tightly bound surfactant (oleic acid) capping, were used as building blocks to form a dense thin inorganic coating on the patterns by a controlled-rate dip coating procedure. The $\mathrm{TiO}_{2}$ NRs served two purposes: $:^{[31,32]}$ first, they further enhanced the hydrophobicity $\left(\mathrm{WCA}_{\mathrm{III}}>\mathrm{WCA}_{\mathrm{II}}\right.$ ) of the micrometer-sized pattern (Scheme 1, panel III), since they superimpose a layer of nanometer-scale roughness with a quite hydrophobic character, due to the NR surfactant capping, onto the organic surface; second, they provided the patterns with a photochemically active coating platform that could progressively change its surface chemical composition from a poorly to a richly hydroxylated state under selected UV illumination $\left(\mathrm{WCA}_{\mathrm{IV}}\right.$ $\left.\ll W_{C A} A_{I I I}\right)$ and revert back upon storage at ambient conditions $^{[31,32]}$ (Scheme 1, panel IV). This hybrid organic-inorganic surface design strategy should ultimately allow the achievement of large reversible excursions between opposite hydrophobic/ hydrophilic wetting regimes (i.e., $\Delta \mathrm{WCA}=\mathrm{WCA}_{\mathrm{III}}-\mathrm{WCA}_{\mathrm{IV}} \gg 1$ ), the extent of which should be purposely controllable by combining suitable surface design and regulating illumination conditions (i.e., time and intensity).

In the following, the validity of our preliminary assumptions is indeed proven by systematic examination of the influence of fabrication parameters and of $\mathrm{TiO}_{2}$ related chemical effects on the wettability dynamics.

The wettability properties of SU-8 patterns were first investigated as a function of their geometric parameters (Scheme 1, panel II). We discuss here structures featured by the same pillar size (42 $\times 42 \mu \mathrm{m}$ in width, $25 \mu \mathrm{m}$ in height), and different inter-pillar spacing, the latter ranging from 14 to $77 \mu \mathrm{m}$. Figure 1 shows representative low-magnification scanning electron microscopy
(SEM) images of three kinds of pillared substrates, along with a photograph of a water droplet laying on the respective surface. The diameter of each droplet is of the order of millimeters and, thus, experiences many periods of each pattern.

The contact values of the water droplets lying on the micropatterned SU-8 substrates are increased compared to that measured for the corresponding flat surface (WCA $\approx 80^{\circ}$ ), and they decrease monotonically from $118.0^{\circ} \pm 1^{\circ}$ to $82.0^{\circ} \pm 1^{\circ}$ as the inter-pillar spacing is enlarged, as demonstrated in Figure 2 (note that the error bars in the figure equal the thickness of the symbols used). In the same figure, this behavior is compared to that predicted by two well-known models-Cassie-Baxters, ${ }^{\text {[51] }}$ and Wenzel' $s^{[52]}$ - that describe the wetting behavior of rough surfaces by invoking different mechanisms.

Cassie-Baxters' model assumes that a water droplet can wet a rough surface only partially, due to the trapping of air underneath the droplet at the recessed regions of the surface. ${ }^{[51]}$ In such circumstances, the contact angle of the liquid droplet, $\theta_{C B}$, can be calculated by the following expression:

$\cos \left(\theta_{\mathrm{CB}}\right)=f_{\mathrm{s}}\left[\cos \left(\theta_{\mathrm{Y}}\right)+1\right]-1$

where $f_{\mathrm{s}}$ is the fraction (always smaller than 1 ) of the solid surface on which the liquid is lying, and $\theta_{\mathrm{Y}}$ is Young's angle, that is, the contact angle of the liquid on the corresponding flat surface with the same chemical characteristics. In our case, $\theta_{\mathrm{Y}}=80^{\circ}$, as found experimentally for a flat SU-8, and $f_{s}$ is calculated according to: ${ }^{[53,54]}$

$$
f_{s}=\frac{1}{(b / a+1)^{2}}
$$

where $a$ is the width and $b$ the spacing of the pillars. Equation 2 is valid for the smooth cutting surface of the pillars. Therefore, a

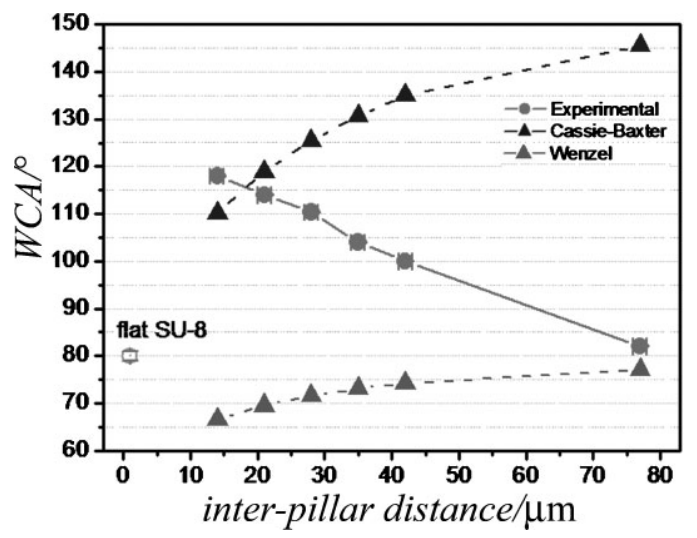

Figure 2. Comparison between WCA values determined experimentally and those calculated theoretically by applying Cassie-Baxters' and Wenzel's models, for the SU- 8 pillared structures $(42 \times 42 \mu \mathrm{m})$ with different inter-pillar distances. The WCA recorded for a flat SU-8 substrate is also reported. 
small divergence from the $f_{\mathrm{s}}$ values calculated by this equation can be expected in the cases of less regular pillars since. Indeed, atomic force microscopy (AFM) measurements reveal that many of the prepared pillar top sides exhibit a characteristic curvature (most often concave in shape), due to the lithographic process itself.

According to Wenzel's model, ${ }^{[52]}$ the water seeps within the recessed regions of the surface, tending to increase the interfacial contact area shared with the solid until a complete wetting condition is eventually achieved. The wettability behavior of a rough surface is then described by the following equation:

$\cos \left(\theta_{\mathrm{W}}\right)=r \cos \left(\theta_{\mathrm{Y}}\right)$

where $\theta_{\mathrm{W}}$ is the value of the contact angle of the liquid on the rough surface, and $r$ is the roughness of the surface, defined as the ratio between the actual area of the rough surface and the geometrically projected area (note that $r$ is always greater than 1 , becoming equal to 1 for flat surfaces). Wenzel's model predicts that when the contact angle of the flat surface is $\theta_{\mathrm{Y}}<90^{\circ}$ (as in the case of our samples), then the WCA of the rough (patterned) surface will be lower than the one of the flat surface. For the pillar geometries used in this work the roughness is defined as: $:^{[53,54]}$

$r=1+\frac{4 f_{s}}{(a / H)}$

where $f_{\mathrm{s}}$ is the same factor as in Cassie-Baxters' model, and $H$ is the height of the pillars. As previously mentioned in the case of Equation 2, Equation 4 also applies to the flat top surfaces of the prepared pillars. Since the pillar-tops most often exhibit a concave curvature and less frequently a convex one, the roughness calculated by Equation 4 is a close approximation to the overall roughness of the surface under examination. In the case of the patterned pillars used herein, an additional approximation is made in the calculation of the WCA following Wenzel's model. Indeed, it is assumed that the water drop lays always on SU-8, whereas a part of it is in contact with the $\mathrm{SiO}_{2} / \mathrm{Si}$ at the bottom of the structures which is actually more hydrophilic than SU-8.

It is clearly inferred from Figure 2 that the monotonic decrease in the measured WCAs from $118.0^{\circ} \pm 1^{\circ}$ to $82.0^{\circ} \pm 1^{\circ}$ for increasingly large inter-pillar spacings, is in contrast with the trend predicted by both theoretical models. The above discrepancy can be rationalized by considering that the wettability response of the pillared microstructures would reflect the occurrence of a gradual transition across different wetting regimes, the latter being dependent on the specific geometries of the involved patterns. On one side, it should be considered that, within the frame of the Cassie-Baxter model, the air fraction of the surface (i.e.; $1-f_{\mathrm{s}}$ ), on which the water droplet is deposited, should scale up with the inter-pillar distances, thereby leading to increased WCA values. On the other hand, upon assuming Wenzel's model, the interfacial contact area between the water droplet and the SU-8 substrate with $\theta_{\mathrm{Y}}<90^{\circ}$ should decrease with increasing the inter-pillar spacing, explaining the increase of the WCA values. Careful examination of our data reveals that the WCA values associated with the SU-8 micrometer-sized patterns with the shortest spacings $(14,21 \mu \mathrm{m})$ are close to the theoretical values predicted by Cassie-Baxters' model. Under such extreme circumstances, the liquid can be reasonably assumed to lay mainly on the top of the pillars without penetrating into the recessed regions. On the contrary, the WCA value measured on the sample characterized by the largest inter-pillar distance $(77 \mu \mathrm{m})$ is close to the value predicted by Wenzel's model, indicating that water is able to wet these types of microstructures completely. Finally, for the patterns with intermediate spacings (28, 35 and $42 \mu \mathrm{m})$, the WCA takes values falling in between those calculated on the basis of the two theoretical models, which suggests that in these cases a partial wetting could actually occur on the microstructured surfaces. In summary, these results allow us reasonably to assume that, as the inter-pillar spacing becomes larger than a critical size threshold (about $\sim 20 \mu \mathrm{m}$ ), the wetting mechanism progressively switches from a Cassie-Baxter to a Wenzel type, crossing an intermediate regime, whereby water starts seeping into the pillars, partially wetting their vertical walls, most likely due to the gravitational forces that become larger than the interfacial tension force between air and water.

Subsequently, the surfaces of the SU-8 patterns were functionalized with a crystalline $\mathrm{TiO}_{2}$ nanomaterial (Scheme 1, panel III) in order, ultimately, to render the wettability of the resulting organic/inorganic hybrid substrates switchable by UV light. For this purpose, we selected single-crystalline anatase $\mathrm{TiO}_{2}$ NRs capped with oleic acid ${ }^{[55]}$ as the building blocks for constructing a compact thin-film coating onto the pillared structures. A representative low-magnification transmission electron microscopy (TEM) image is shown in Figure 3a, which demonstrates that the colloidal samples consist of unidirectionally elongated uniform nanoparticles with a rod-like profile whose average short- and long-axis dimensions are of $\sim 3 \mathrm{~nm}$ and $\sim 20$ $25 \mathrm{~nm}$, respectively. As discussed elsewhere, ${ }^{[31,55]}$ such $\mathrm{TiO}_{2} \mathrm{NRs}$ are characterized by a preferential elongation along the $c$-axis of
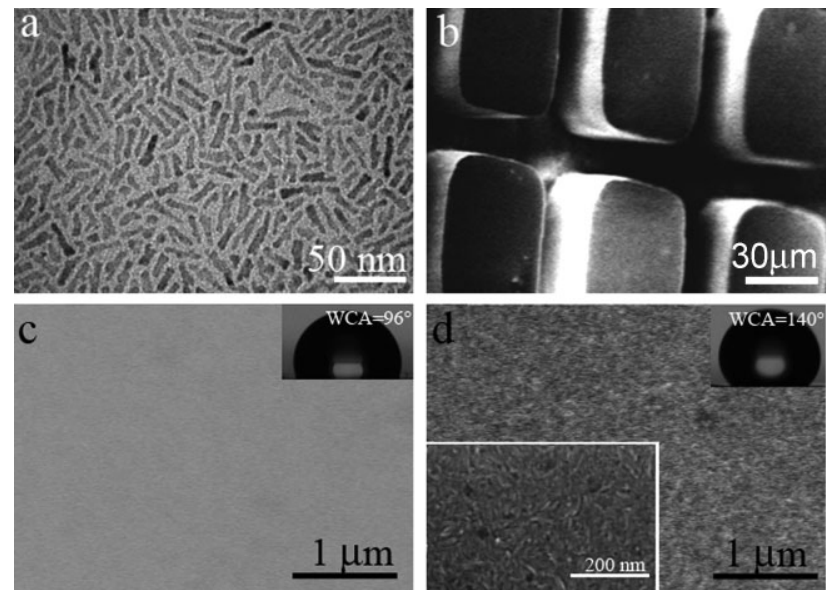

Figure 3. a) Low-magnification TEM image of the as-synthesized $3 \times 20 \mathrm{~nm} \mathrm{TiO}{ }_{2}$ nanorods. b) Low-resolution SEM image of $21-\mu \mathrm{m}$-spaced $\mathrm{TiO}_{2}$-coated SU-8 pillars, collected at a $45^{\circ}$ tilt angle. c and d) Highmagnification SEM images of a bare and $\mathrm{TiO}_{2}$-nanorod-coated top surface of a pillar, respectively. In the corresponding top-right insets, the optical images of a water droplet in contact with such surfaces are shown for the respective cases. It is apparent that upon $\mathrm{TiO}_{2}$ coating the substrates acquire enhanced hydrophobic character. 
the anatase lattice, and expose stepped longitudinal sidewalls dominantly made of crystallographically equivalent $(011) /(101)$ facets, whereas their apexes terminate exclusively with (001) facets. As a primary practical advantage, such nanocrystals are entirely processable in the liquid phase, so that they can be easily deposited onto any desired substrate from their optically clear nonpolar solution by an inexpensive and simple controlled-rate dipcoating technique (see the Experimental Section). Figure 3b-d reports typical results of SEM inspection performed on SU-8 patterns (with a $21-\mu \mathrm{m}$ inter-pillar spacing) before and after the application of the $\mathrm{TiO}_{2}$ deposition procedure. The low-magnification overview taken at a $45^{\circ}$ tilt angle in Figure $3 \mathrm{~b}$ reveals preservation of the initial flat morphology of the top sides of the pillars, which preliminarily indicates that the employed dipcoating technique guarantees deposition of a uniform nanocrystal layer onto the patterns without inducing any noticeable formation of sub-micron particle aggregates. Spontaneous adhesion of the surfactant-passivated NRs to the patterned surfaces should be promoted by the inherent character of the available types of surfaces. The higher degree of hydrophobicity of the exposed surfaces of the prominent SU-8 pillars (i.e., the top and lateral sidewalls) relative to that of the bottom polymer-free areas of the underlying $\mathrm{SiO}_{2} / \mathrm{Si}$ wafer (the WCAs on the corresponding smooth materials are about $80^{\circ}$ and $50^{\circ}$, respectively) is expected to result in a denser $\mathrm{TiO}_{2}$ coverage on the former rather than the latter regions. This is indeed proven by high-magnification SEM inspection of the top surfaces of the pillars. Comparative examination of Figure $3 c-d$ clearly confirms that a continuous and crack-free NR coverage is achieved on such regions over areas as large as several squared micrometers. The compact films enclose tightly packed arrays of NRs, most of which are preferentially accommodated in a roughly parallel orientation with respect to the substrate underneath. The self assembly of the NRs in such types of densely laterally arranged superstructures is promoted by the concurrent effects of the anisotropic shape of the nanocrystals and of the surfactant capping at their surface.

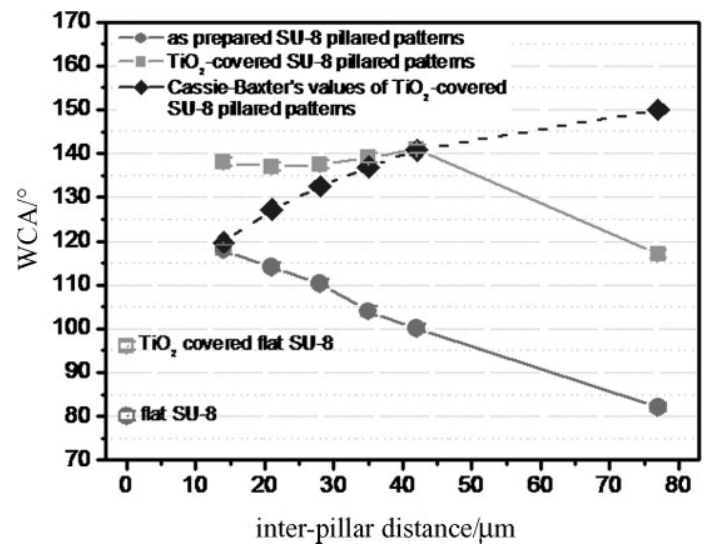

Figure 4. Experimental WCA values for the SU-8 pillared $(42 \times 42 \mu \mathrm{m})$ microstructures before (circles), and after $\mathrm{TiO}_{2}$ coating (squares), respectively, and theoretical WCA values for $\mathrm{TiO}_{2}$-coated pillars calculated following the Cassie-Baxter model (diamonds) as a function of the inter-pillar spacing. The WCA values recorded for an uncoated and $\mathrm{TiO}_{2}$-coated flat SU-8 substrate are also reported. The error bars in the figure are equal to the thickness of the used symbols.
During solvent evaporation, the NRs tend to strengthen the hydrophobic interactions among each other as a means of decreasing the overall surface energy of the system. ${ }^{[31,32]}$

As demonstrated in the insets of Figure $3 c$ and $d$ and in Figure 4, the $\mathrm{TiO}_{2}$ coating attained has a remarkable impact on the wettability response of the SU-8 structures, which indeed exhibit enhanced hydrophobicity, as compared to the corresponding bare polymer surfaces (Scheme 1, panel III). Such modified properties should reasonably result from the contribution of the additional nanometer-sized roughness that the $\mathrm{TiO}_{2} \mathrm{NRs}$, which expose the hydrophobic long alkyl chain of the capping surfactants, induce onto the SU-8 surfaces because of their nanometer-scale dimensions and peculiar packing arrangement. It is worth mentioning here that the patterning process does not introduce any additional nanometer-sized roughness to the SU-8 surfaces before the functionalization with the $\mathrm{TiO}_{2} \mathrm{NRs}$. Notably, the increase in WCA measured after NR addition ranges from about $20^{\circ}$ up to $40^{\circ}$ for patterns with progressively larger interpillar distances, which suggests a clear synergy between the micro- and nanoscale surface structuring in the modification of the wetting properties of the $\mathrm{TiO}_{2}$-functionalized substrates. In Figure 4 a comparison is also shown between the WCAs measured on the NR-covered pillars and the theoretical values predicted by the Cassie-Baxter model. By using the WCA measured on the $\mathrm{TiO}_{2}$-covered flat SU-8 surface, $\theta_{\mathrm{Y}}=96^{\circ}$, as the Young's angle in Equation 1, it is demonstrated that the experimental values are greater than the theoretical ones, except from the value measured on the structure with the largest spacing of $77 \mu \mathrm{m}$. Therefore, with the exception of the $77-\mu \mathrm{m}$ inter-pillar spacing, we can safely assume that the water droplets lay explicitly on the top of the patterned pillars after their coverage with the $\mathrm{TiO}_{2}$ NRs. The dual-scale roughness achieved appears to enable the trapping of more numerous air pockets underneath the water droplet, thus preventing the liquid from seeping into the void spaces among the pillars. This effect is similar to that observed on lotus leaves, on which the association of micro-/nanoscale morphological features is ultimately responsible for their superhydrophobic properties. ${ }^{[56,57]}$

The mismatch between the experimental and the theoretical WCA values on the NR-functionalized SU-8 pillars can plausibly be ascribed to the attainment of an enhanced degree of nanometer-sized roughness on the SU-8 micropillars compared to the one achieved on the flat SU-8 after the dipping procedure. Such a hypothesis implies that the use of the WCA obtained on the NR-covered flat SU-8 as the Young angle $\left(\theta_{\mathrm{Y}}=96^{\circ}\right)$ in Equation 1 would introduce an error in the theoretical calculations, since in Cassie-Baxters' picture the Young's angle is defined as the WCA measured on the corresponding flat surface possessing the same physicochemical characteristics. Instead, the actual Young's angle could exhibit a higher value due to the increased surface nanometer-sized roughness.

To check the validity of the above assumption, we used AFM. Indeed, the measurements ${ }^{[6,58,59]}$ of $\mathrm{TiO}_{2}$-covered substrates showed enhanced roughness in the case of the micropillars with respect to the flat SU-8 sample. The root mean square (RMS) of the distribution of height values in the AFM images was taken as an estimation of the roughness. In Table 1 , the average values of RMS are reported for the flat and patterned SU-8 surfaces coated with $\mathrm{TiO}_{2}$ NRs. For the pillared SU-8 substrates the RMS values 
Table 1. Surface roughness calculated on the basis of $41 \times 41-\mu \mathrm{m}$ areas scanned by AFM on NR-coated (both flat and micropillared) SU-8 surfaces. The overall RMS embodies the contributions from both nanometer-sized roughness and waviness of the surface, whereas the high-frequency RMS represents only nanometer-sized roughness, after waviness background removal.

\begin{tabular}{lcc}
\hline 41- $\mu$ m-wide AFM scan & $\begin{array}{c}\text { Overall } \\
\text { RMS [nm] }\end{array}$ & $\begin{array}{c}\text { High-frequency RMS } \\
\text { (nanometer-sized roughness) [nm] }\end{array}$ \\
\hline $\mathrm{TiO}_{2}$ NR-coated flat SU-8 & $10.0 \pm 0.5$ & $9.8 \pm 0.5$ \\
$\mathrm{TiO}_{2}$ NR-coated pillared SU-8 & $67 \pm 15$ & $38 \pm 10$
\end{tabular}

do not change significantly with the inter-pillar spacing and are quite similar for all the pillar geometries examined. The overall RMS embodies the contributions from both the nanometer-sized roughness (high frequency RMS) and waviness (low frequency RMS) of the surface, whereas the high-frequency RMS, extracted after subtraction of the wavy background, reflects the nanometersized roughness only (see the Experimental Section). For the NRcoated flat SU-8, the overall RMS and the high-frequency RMS are identical. In contrast, for the NR-coated SU-8 pillared patterns, the overall RMS is almost twice as much as the high-frequency RMS component only. Indeed, the pillar-top sides exhibit a characteristic curvature (most often concave in shape), which is believed to be imparted by the lithographic process itself. Interestingly, even after removal of such (low-frequency) waviness, the residual nanometer-sized roughness (highfrequency RMS) of the NR-coated pillared samples is still four times as high as that found for the NR-coated flat SU-8 samples. Such values may be explained by considering that the curvature of the top surfaces of the pillars may disturb the process of nanocrystal self assembly during the solvent evaporation stage, eventually causing a relatively larger fraction of NRs to be deposited and trapped under unparallel orientations with respect to the substrate underneath. It turns out that such NRs protruding out of the attained nanocrystal thin film could actually impart a higher degree of nanometer-sized roughness to the top surfaces of the pillars, as compared to that generally observed for NR-based films achieved on flat polymer substrates. ${ }^{[31]}$ Therefore, in agreement with our preliminary hypothesis, it can be concluded that the discrepancy between the experimental WCA values and the ones calculated by the Cassie-Baxter equation is due to the nonequivalence of the inherent physicochemical surface properties of the $\mathrm{TiO}_{2}$-based coatings when they are formed on the flat or on the micropatterned SU-8 substrates, respectively.

Finally, the samples were exposed to pulsed UV laser light irradiation to verify the possibility of controllably changing the wettability properties of $\mathrm{TiO}_{2}$-coated SU-8 patterns in a reversible manner on the basis of the light-driven hydrophilization mechanism of $\mathrm{TiO}_{2}$ surfaces (Scheme 1, panel IV). This conversion is generally believed to be initiated by photogenerated holes that create oxygen vacancies at the semiconductor surface. These defects are then able to promote dissociative adsorption of atmospheric water, which results in an increased degree of surface hydroxylation. The coordinated hydroxyl groups promote the adhesion of further water molecule multilayers, making the surfaces highly hydrophilic.

For the hybrid organic/inorganic patterns here, the organized NR arrays onto the SU-8 surfaces should preferentially expose (011)/(101) anatase facets that are particularly effective in the light-induced hydrophilization mechanism of $\mathrm{TiO}_{2}{ }^{[20-32]}$ In addition, under the specific irradiation conditions used in this work (see the Experimental Section), a remarkable increase in hydrophilicity can be observed, which is correlated with the increase in the degree of surface hydroxylation of $\mathrm{TiO}_{2}$, while the surfactant molecules anchored to the NR surfaces are not subjected to noticeable photocatalytic degradation. ${ }^{[31,32]}$ Therefore, the porous $\mathrm{TiO}_{2}$ NRs-film is expected to embody a distribution of alternating highly hydrophilic and quite hydrophobic domains, the former being related to the newly introduced -OH groups, and the latter being associated with surfactantprotected $\mathrm{TiO}_{2}$ areas. Such surface arrangements could lead to nanometer-sized capillary infiltration, allowing water droplets to spread out over such $\mathrm{TiO}_{2}$-based coatings. ${ }^{[31]}$ This mechanism ultimately ensures the highest wettability excursion to be achieved with a net illumination time of only $0.216 \mathrm{~ms}$, and guarantees full recovery of the initial hydrophobic state in the dark. ${ }^{[31,32]}$

We calculated the surface energy of our samples, before and after the introduction of the $\mathrm{TiO}_{2}$ NRs on the SU-8 pillars and after UV irradiation of the covered pillars, in order to quantify the variations in its polar component. The calculation of the surface energies is based on the equation introduced by Owens and Wendt, ${ }^{[60]}$ using a particular piece of software. The surface tension $\sigma$ is separated into polar and dispersive components, $\sigma_{\mathrm{P}}$ and $\sigma_{\mathrm{D}}$, respectively, where the polar part describes the hydrophilic character and the dispersive part the hydrophobic character of the surface:

$\sigma=\sigma_{\mathrm{P}}+\sigma_{\mathrm{D}}$

In our measurements, two liquids were used: water and di-iodomethane. The values of the dispersive and polar components of the surface tensions are shown in Table 2. An increase in the surface energies of the $\mathrm{TiO}_{2}$-coated surface can clearly be observed after UV irradiation. As expected, it can be verified that the surface coated with $\mathrm{TiO}_{2}$ before UV irradiation exhibits a very small polar component. However, after UV irradiation, the polar share of

Table 2. Surface energy between SU-8 surface coated with $\mathrm{TiO}_{2}$ before and after irradiation, with the dispersive part and the polar part of the surface energy, respectively.

\begin{tabular}{lcc}
\hline Surface & Surface tensions $\left[\mathrm{mN} \mathrm{m}^{-1}\right]$ & Dispersive component $\left[\mathrm{mN} \mathrm{m}^{-1}\right]$ \\
\hline SU-8 & 38.23 & Polar component $\left[\mathrm{mN} \mathrm{m}^{-1}\right]$ \\
Surface coated with $\mathrm{TiO}_{2}$ before UV irradiation & 35.45 & 1.76 \\
Surface coated with $\mathrm{TiO}_{2}$ after UV irradiation & 72.18 & 35.38 \\
\hline
\end{tabular}



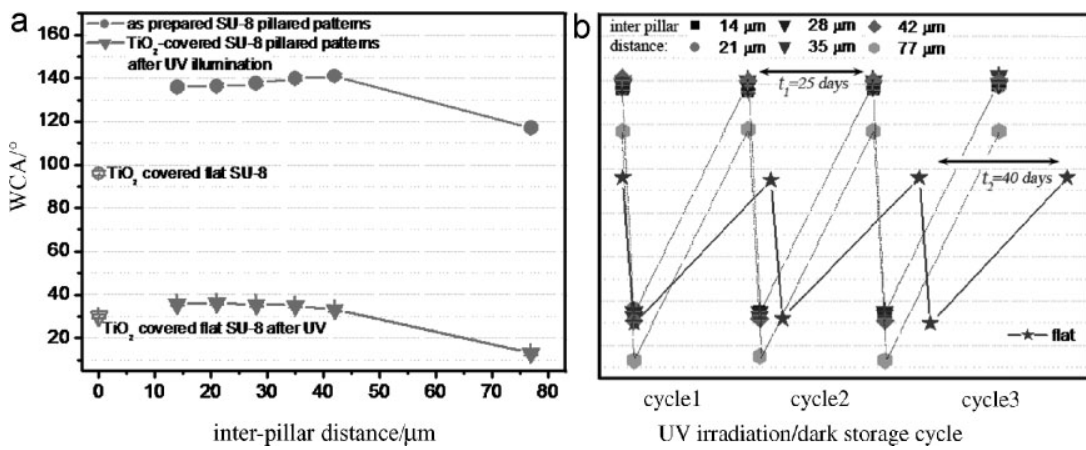

Figure 5. a) Experimental WCA values for the SU-8 pillared $(42 \times 42 \mu \mathrm{m})$ microstructures covered with $\mathrm{TiO}_{2} \mathrm{NRs}$ before any irradiation (circles), and after a subsequent 120 min of pulsed UV irradiation (triangles), as a function of the inter-pillar distance (note that an overall $120 \mathrm{~min}$ duration of the pulsed UV experiment corresponded to an actual irradiation time of $0.216 \mathrm{~ms}$ ). The WCA values recorded for the $\mathrm{TiO}_{2}$-coated flat SU-8 substrate before and after irradiation are also reported. b) Reversible wettability changes during cyclic alternations of 120-min pulsed UV illumination, and 40- or 20-day storage in the dark under ambient conditions for the flat and the patterned SU-8 respectively. Note that the lines connecting the values of the WCA in (b) are just guides for the eye and are not related to any temporal evolution within the relevant cycles.

the surface energy assumes a significant component itself in relation to the total surface energy. Indeed, Figure 5a demonstrates that, upon UV irradiation, the $\mathrm{TiO}_{2}$-coated $\mathrm{SU}-8$ patterns exhibit a very hydrophilic character (WCA $\approx 15-30^{\circ}$ ), with a decrease in the WCA values by more than $100^{\circ}$ (the error bars in the figure are within the thickness of the symbols). It is worth remarking that the light-driven WCA excursions measured on the micropillared structures are larger than those found for the corresponding flat substrate $\left(\Delta \mathrm{WCA} \approx 65^{\circ}\right)$, demonstrating the beneficial effect of the micrometer-scale texturing on the ultimate degree of hydrophilicity achievable. In this respect, it is conceivable that the droplet spreading over the substrate is facilitated by its front experiencing the highly hydrophilic $\mathrm{TiO}_{2}$ film located both on the top surfaces and on the sidewalls of the SU-8 pillars. The WCA values are expected to be smaller for narrower inter-pillar spacing, since the interfacial area of contact between the drop and the highly hydrophilic surfaces is larger. Nevertheless, the results presented in Figure 5a show that the lower WCA after UV irradiation of the surfaces is achieved for the pillar arrangement with the larger spacing. A plausible explanation could be that comparatively large spacing between the SU-8 structures may facilitate functionalization with a uniform $\mathrm{TiO}_{2}$ films on both the top and the sidewalls of the SU-8 pillars, as well as on the recessed $\mathrm{SiO}_{2} / \mathrm{Si}$ regions. On the contrary, as the interpillar spacing is reduced, it is likely that the process of nanocrystal self organization in continuous films may be perturbed, resulting in more sparsely distributed $\mathrm{TiO}_{2}$ island-like films at the sides and the bottom of the pillars. Thus, it can be expected that, after UV irradiation of the different pillar arrangements, the most hydrophilic surfaces should actually be those that have been covered with a more densely and uniformly packed $\mathrm{TiO}_{2} \mathrm{NR}$ coating, that is, the larger-spaced pillars.

As reported previously, ${ }^{[20-32]}$ the $\mathrm{OH}$ groups newly implanted onto the $\mathrm{TiO}_{2}$ surfaces upon illumination are neither thermodynamically nor kinetically stable. In fact, once UV irradiation is halted, $\mathrm{O}_{2}$ substitution for the newly implanted hydroxyl moieties becomes the prevalent chemical reaction under air in the dark, leading to progressive attenuation of the hydrophilicity gained under light. Figure $5 b$ confirms that, subsequent to the light stimulation, during prolonged storage of the samples in the dark under ambient conditions they revert back to their initial hydrophobic conditions, close to those encountered in freshly prepared $\mathrm{TiO}_{2}$-coated $\mathrm{SU}-8$ substrates. Interestingly, the average recovery time is generally faster for the micropatterned samples (from the figure: $t_{1} \approx 25$ days) than for the flat surface counterpart (from the figure: $t_{2} \approx 40$ days), which presumably indicates easier access of atmospheric oxygen to all the substrate regions functionalized with the $\mathrm{TiO}_{2}$ NRs. The process of reversibly changing the WCA can be carried out over several cycles of irradiation/dark storage, proving that the NR coatings exhibit excellent reversibility in their wettability properties without being affected by any significant photochemical fatigue. Work is in progress to understand the dynamics of the recovery of the samples towards their hydrophobic state, as well as to control the time of the recovery using external stimuli.

\section{Conclusions}

We have studied the wettability response of purposely engineered solid substrates with dual-scale surface texturing, which consist of photolithographically patterned SU-8 micropillars covered by $\mathrm{TiO}_{2}$ nanorods. The as-derived hybrid organic/inorganic structures exhibit a highly hydrophobic character due to the combination of the microroughness of the SU-8 pillars with the nanometer-sized roughness of the surfactant-passivated nanorods coating. The system changes its wettability upon pulsed UV laser irradiation into a highly hydrophilic state, in which water is allowed to seep into the recessed regions among the pillars. The change is primarily due to the increased hydroxylation degree of the $\mathrm{TiO}_{2} \mathrm{NR}$ films upon irradiation with UV light. The ultimate wettability excursions achievable are as large as about $100^{\circ}$ due to the micro-/nanoscale amplification effect. The hybrid structures can fully recover their initial hydrophobicity after a few weeks upon storage in the dark in air. The reversible wetting behavior of the hybrid surfaces can be repeated without any apparent fatigue upon successive cycles of alternated UV irradiation and dark storage.

The present work highlights that a combination of micrometerscale patterning in polymeric substrates and nanometer-scale roughness introduced by nanocrystals of light sensitive semiconductor oxides is a key factor in enhancing the switchable wettability of artificial surfaces over a broad WCA range. By an appropriate choice of geometry and control over the wettability switching time, it should be possible to devise substrates exhibiting challenging capabilities in the realization of smart liquid responsive surfaces and microfluidic reactors operating under external light stimulation. Indeed, this work presents a systematic study towards the understanding of the mechanisms and the geometrical features needed for the realization of such 
systems. The pillar geometries were chosen on the basis of their radial symmetry essential for enabling WCA data analysis with existing theoretical models. Among other polymers, SU-8 was selected because of its high processability, elevated mechanical toughness, and chemical resistance, characteristics that make it a promising candidate for microfluidic systems.

\section{Experimental}

Materials: All chemicals were of the highest purity available and were used as received. Titanium tetraisopropoxide [Ti(OPri $)_{4}$ or TTIP, $\left.97 \%\right]$, trimethylamine $\mathrm{N}$-oxide dihydrate $\left[\left(\mathrm{CH}_{3}\right)_{3} \mathrm{NO} \cdot 2 \mathrm{H}_{2} \mathrm{O}\right.$ or TMAO, 98\%] and oleic acid $\left(\mathrm{C}_{17} \mathrm{H}_{33} \mathrm{CO}_{2} \mathrm{H}\right.$ or OLAC, $\left.90 \%\right)$ were purchased from Aldrich. Silicon wafers were purchased from Jocam. SU-8 2010 was purchased from MicroChem. All solvents used were of analytical grade and were also purchased from Aldrich. Water was bidistilled (Millipore Q).

Preparation of SU-8 Masters: SU-8 micropatterns were prepared on silicon wafer substrates by photolithography. Due to the formation of a ubiquitous oxide surface layer under ambient conditions, the substrates are henceforth referred to as $\mathrm{SiO}_{2} / \mathrm{Si}$. First, the substrates were cleaned by ultrasonication in acetone for $10 \mathrm{~min}$ and in propan-2-ol for $10 \mathrm{~min}$, after which they were dried under a nitrogen airflow. SU-8 was processed according to a procedure reported in the manual of MicroChem Corp. [61]. Each substrate was spin-coated with the polymer in order to obtain a desired thickness of about $25 \mu \mathrm{m}$. The patterning was carried out by using a Karl Suss MJ B3 mask-aligner with UV illumination and a photomask containing the patterns. Post-exposure bake (PEB) was performed on a hot plate to achieve ultimate crosslinking of the resist, which was then allowed to cool down in order to improve adhesion of SU-8 to the substrate. The wafers were then developed by immerging them into a SU-8 developer and propan-2-ol.

Synthesis of $\mathrm{TiO}_{2}$ Nanorods (NRs): The nanocrystal synthesis was carried out under nitrogen flow using a standard Schlenk line set-up. Anatase $\mathrm{TiO}_{2}$ nanorods (NRs) with an average diameter of $\sim 3-4 \mathrm{~nm}$ and a mean length of $\sim 20-25 \mathrm{~nm}$ were obtained by low-temperature TMAOcatalyzed hydrolysis of TTIP [55]. Typically, $10 \mathrm{mmol}$ of TTIP was dissolved in $70 \mathrm{~g}$ of degassed oleic acid (OLAC) and the resulting solution was then reacted with $3.5 \mathrm{~mL}$ of an aqueous $2 \mathrm{M} \mathrm{TMAO}$ solution at $100^{\circ} \mathrm{C}$ for $72 \mathrm{~h}$. The $\mathrm{TiO}_{2} \mathrm{NRs}$ were separated from their growing mixture upon propan-2-ol addition and were subsequently subjected to repeated cycles of redissolution in toluene and precipitation with acetone to wash out surfactant residuals. Finally, optically clear $\mathrm{TiO}_{2}$ stock solutions in toluene were prepared and stored under ambient conditions.

Preparation of $\mathrm{TiO}_{2}$ Film Coatings on the SU-8 Patterns: The $\mathrm{TiO}_{2} \mathrm{NR}$ coatings were fabricated under ambient laboratory conditions as follows. The SU-8 patterns were dipped into a $0.4 \mathrm{M}$ (expressed in $\mathrm{TiO}_{2}$ molecular units, according to ICP-AES analysis) toluene $\mathrm{TiO}_{2}$ solution, using a dip coater system (NIMA, Nima Technology, Coventry). The sample was dipped up to fifty times and withdrawn with a rate of $1 \mathrm{~cm} \mathrm{~min}^{-1}$. The asprepare films were gently dried with a nitrogen flow and stored at ambient conditions.

Irradiation Experiments: The $\mathrm{TiO}_{2}$ coatings were irradiated with the third harmonic wavelength $(355 \mathrm{~nm})$ of a pulsed Nd-YAG laser, with pulse duration of $3 \mathrm{~ns}$, a repetition rate of $10 \mathrm{~Hz}$, and energy density of $5 \mathrm{~mJ} \mathrm{~cm}{ }^{-2}$. The total duration of the irradiation experiments, which was required to detect the largest wettability changes, was found to be $120 \mathrm{~min}$. This period corresponds to 72000 laser pulses and, hence, to an actual interaction time of UV photons with the $\mathrm{TiO}_{2}$ samples of only $0.216 \mathrm{~ms}$. Additional irradiation did not influence the wettability of the prepared films any further.

Elemental Analysis: The Ti atomic content of the nanocrystal solution was measured by inductively coupled plasma atomic emission spectroscopy (ICP-AES) measurements with a Varian Vista AX spectrometer. The samples for analyses were digested in concentrated $\mathrm{HCl} / \mathrm{HNO}_{3}(3 / 1 \mathrm{v} / \mathrm{v})$.

Contact Angle Measurements: Contact angle characterization was performed on the substrates by the sessile drop method using a
CAM200-KSV instrument (equipped with a digital camera for acquiring magnified images of the microdroplets). Bidistilled water was used for these tests and dispensed by means of a microsyringe. Typically, the droplet volume was $3 \mu \mathrm{L}$. The WCA value was the average of 10 measurements recorded on different neighboring surface locations. The estimated error on a single measurement was $\pm 1^{\circ}$. Measurements were carried out on uncoated and $\mathrm{TiO}_{2}$-coated substrates before irradiation and immediately after subjecting the samples to a known number of laser pulses, as well as after storing the samples in the dark under ambient conditions (relative humidity $30-40 \%)$.

Transmission Electron Microscopy (TEM): Low-magnification TEM images of $\mathrm{TiO}_{2}$ NRs were recorded with a Jeol Jem 1011 microscope operating at an accelerating voltage of $100 \mathrm{kV}$. The samples for TEM analyses were prepared by dropping a dilute solution of the NRs dissolved in toluene or chloroform onto carbon-coated copper grids and then allowing the solvent to evaporate.

Scanning Electron Microscopy (SEM): Low-resolution SEM characterization of $\mathrm{TiO}_{2}$ coatings deposited onto silicon substrates was performed with a RAITH 150 EBL instrument. Typically, the images were acquired at low accelerating voltages (less than $5 \mathrm{kV}$ ) using short exposure times.

Atomic Force Microscopy (AFM): AFM measurements were carried out using an Asylum Research MFP3D instrument, operating in contact mode with standard silicon probes CSG10 (NT-MDT). The nominal tip apex diameter was $\sim 10 \mathrm{~nm}$, the tip length was $\sim 4 \mu \mathrm{m}$, and the nominal cantilever spring constant was $\sim 0.1 \mathrm{~N} \mathrm{~m}^{-1}$. As the pillar height $(H=25 \mu \mathrm{m})$ was larger than the Z-range of the AFM actuator $(\sim 15 \mu \mathrm{m})$ and much larger than the tip height $(\sim 4 \mu \mathrm{m})$, it was not possible to track the pillar top surfaces in constant force mode, under which the probes, in fact, were frequently broken. Instead, the AFM contact mode was operated in constant height, and the deflection was used to extract the topography of the topmost pillars, after proper calibration of the optical-lever detection system. Removal of the low-frequency RMS (i.e., the waviness) from the AFM images was performed by subtracting the best fitting second-order polynomial surface, instead of the standard plane subtraction (first-order polynomial).

\section{Acknowledgements}

G. Caputo and B. Cortese contributed equally to this work. This work was partially supported by the Italian Ministry of Research (contract no. RBIN048TSE).

Received: July 3, 2008

Revised: November 3, 2008

Published online: March 9, 2009

[1] X. J. Feng, L. Jiang, Adv. Mater. 2006, 18, 3063.

[2] J. X. Wang, Y. Q. Wen, J. P. Hu, Y. L. Song, L. Jiang, Adv. Func. Mater. 2007, $17,219$.

[3] F. C. Cebeci, Z. Z. Wu, L. Zhai, R. E. Cohen, M. F. Rubner, Langmuir 2006, 22, 2856.

[4] R. P. Garrod, L. G. Harris, W. C. E. Schofield, J. McGettrick, L. J. Ward, D. O. H. Teare, J. P. S. Badyal, Langmuir 2007, 23, 689.

[5] Y. Masuda, K. Kato, Chem. Mater. 2008, 20, 1057.

[6] D. Lee, M. F. Rubner, R. E. Cohen, Nano Lett. 2006, 6, 2305.

[7] K. Tadanaga, J. Morinaga, A. Matsuda, T. Minami, Chem. Mater. 2000, 12, 590.

[8] S. T. Wang, L. Feng, H. Liu, T. L. Sun, X. Zhang, L. Jiang, D. B. Zhu, ChemPhysChem 2005, 6, 1475.

[9] H. Notsu, W. Kubo, I. Shitanda, T. Tatsuma, J. Mater. Chem. 2005, 15, 1523.

[10] E. Hosono, H. Matsuda, I. Honma, M. Ichihara, H. Zhou, Langmuir 2007, 23, 7447.

[11] K. Teshima, H. Sugimura, A. Takano, Y. Inoue, O. Takai, Chem. Vap. Depos. 2005, 11, 347. 
[12] H. S. Lim, J. T. Han, D. Kwak, M. Jin, K. Cho, J. Am. Chem. Soc. 2006, 128, 14458.

[13] A. Athanassiou, M. I. Lygeraki, D. Pisignano, K. Lakiotaki, M. Varda, E. Mele, C. Fotakis, R. Cingolani, S. H. Anastasiadis, Langmuir 2006, 22, 2329.

[14] A. Athanassiou, M. Varda, E. Mele, M. I. Lygeraki, D. Pisignano, M. Farsari, C. Fotakis, R. Cingolani, S. H. Anastasiadis, Appl. Phys. A 2006, 83, 351.

[15] E. Mele, D. Pisignano, M. Varda, M. Farsari, G. Filippidis, C. Fotakis, A. Athanassiou, R. Cingolani, Appl. Phys. Lett. 2006, 88, 203124.

[16] M. I. Lygeraki, E. Tsiranidou, S. H. Anastasiadis, C. Fotakis, D. Pisignano, R. Cingolani, A. Athanassiou, Appl. Phys. A 2008, 91, 397.

[17] X. Yu, Z. Q. Wang, Y. G. Jiang, F. Shi, X. Zhang, Adv. Mater. 2005, 17, 1289.

[18] L. B. Xu, W. Chen, A. Mulchandani, Y. S. Yan, Angew. Chem. Int. Ed. 2005, $44,6009$.

[19] T. L. Sun, G. J. Wang, L. Feng, B. Q. Liu, Y. M. Ma, L. Jiang, D. B. Zhu, Angew. Chem. Int. Ed. 2004, 43, 357.

[20] R. Wang, K. Hashimoto, A. Fujishima, M. Chikuni, E. Kojima, A. Kitamura, M. Shimohigoshi, T. Watanabe, Nature 1997, 388, 431.

[21] R. Wang, K. Hashimoto, A. Fujishima, M. Chikuni, E. Kojima, A. Kitamura, M. Shimohigoshi, T. Watanabe, Adv. Mater. 1998, 10, 135.

[22] T. L. Thompson, J. T. Yates, Chem. Rev. 2006, 106, 4428.

[23] N. Sakai, A. Fujishima, T. Watanabe, K. Hashimoto, J. Phys. Chem. B 2001, 105, 3023

[24] A. Nakajima, S. Koizumi, T. Watanabe, K. Hashimoto, Langmuir 2000, 16, 7048.

[25] N. Sakai, A. Fujishima, T. Watanabe, K. Hashimoto, J. Phys. Chem. B 2003, 107, 1028.

[26] M. Miyauchi, A. Nakajima, A. Fujishima, K. Hashimoto, T. Watanabe, Chem. Mater. 2000, 12, 3.

[27] X. J. Feng, L. Feng, M. H. Jin, J. Zhai, L. Jiang, D. B. Zhu, J. Am. Chem. Soc. 2004, 126, 62.

[28] X. J. Feng, J. Zhai, L. Jiang, Angew. Chem. Int. Ed. 2005, 44, 5115.

[29] S. T. Wang, X. J. Feng, J. N. Yao, L. Jiang, Angew. Chem. Int. Ed. 2006, 45, 1264.

[30] H. S. Lim, D. Kwak, D. Y. Lee, S. G. Lee, K. Cho, J. Am. Chem. Soc. 2007, 129, 4128.

[31] G. Caputo, C. Nobile, T. Kipp, L. Blasi, V. Grillo, E. Carlino, L. Manna, R. Cingolani, P. D. Cozzoli, A. Athanassiou, J. Phys. Chem. C 2008, 112, 701.

[32] G. Caputo, C. Nobile, R. Buonsanti, T. Kipp, L. Manna, R. Cingolani, P. D. Cozzoli, A. Athanassiou, J. Mater. Sci. 2008, 43, 3474.

[33] N. Stevens, C. I. Priest, R. Sedev, J. Ralston, Langmuir 2003, 19, 3272.

[34] Y. Tian, H. Notsu, T. Tatsuma, Photochem. Photobiol. Sci. 2005, 4, 598.

[35] X. T. Zhang, M. Jin, Z. Y. Liu, D. A. Tryk, S. Nishimoto, T. Murakami, A. Fujishima, J. Phys. Chem. C 2007, 111, 14521.
[36] X. T. Zhang, O. Sato, A. Fujishima, Langmuir 2004, 20, 6065

[37] T. Tatsuma, W. Kubo, A. Fujishima, Langmuir 2002, 18, 9632.

[38] W. Kubo, T. Tatsuma, A. Fujishima, H. Kobayashi, J. Phys. Chem. B 2004 108,3005

[39] W. Kubo, T. Tatsuma, Appl. Surf. Sci. 2005, 243, 125.

[40] A. Kanta, R. Sedev, J. Ralston, Langmuir 2005, 21, 5790.

[41] N. Saito, H. Haneda, K. Koumoto, Microelectron. J. 2004, 35, 349.

[42] Y. W. Koh, M. Lin, C. K. Tan, Y. L. Foo, K. P. Loh, J. Phys. Chem. B 2004, 108 11419.

[43] O. Azzaroni, P. L. Schilardi, R. C. Salvarezza, J. Manuel-Herrero, C. Zaldo, L. Vázquez, Appl. Phys. A 2005, 81, 1113

[44] D. Palms, C. Priest, R. Sedev, J. Ralston, G. Wegner, J. Colloid Interface Sci. 2006, 303, 333

[45] H. Shao, X. F. Qian, B. C. Huang, Mater. Sci. Semicond. Process. 2007, 10 68.

[46] Y. Masuda, K. Kato, Chem. Mater. 2008, 20, 1057.

[47] D. Chen, Y. Gao, G. Wang, H. Zhang, W. Lu, J. Li, J. Phys. Chem. C 2007, $111,13163$.

[48] Y. Xu, X. Zhu, Y. Dan, J. H. Moon, V. W. Chen, A. T. Johnson, J. W. Perry, S. Yang, Chem. Mater. 2008, 20, 1816.

[49] P. Kern, Y. Muller, J. Patscheider, J. Michler, J. Phys. Chem. B 2006, 110 23660.

[50] a) M. H. Park, Y. J. Jang, H. M. Sung-Suh, M. M. Sung, Langmuir 2004, 20 2257. b) S. Natarajan, D. A. Chang-Yen, B. K. Gale, J. Micromech. Microeng. 2008, 18, 045021 . c) S. A. Soper, S. M. Ford, S. Qi, R. L. McCarley, K. Kelly, M. C. Murphy, Anal. Chem. 2000, 72, 642. d) J. Zhang, M. B. Chan-Park, S. R. Conner, Lab Chip 2004, 4, 646.

[51] A. B. D. Cassie, S. Baxter, Trans. Faraday Soc. 1944, 40, 546.

[52] R. N. Wenzel, Ind. Eng. Chem. 1936, 28, 988.

[53] L. Zhu, Y. Y. Feng, X. Y. Ye, Z. Y. Zhou, Sens. Actuators A 2006, 130-131, 595.

[54] R. D. Narhe, D. A. Beysens, Langmuir 2007, 23, 6486.

[55] P. D. Cozzoli, A. Kornowski, H. Weller, J. Am. Chem. Soc. 2003, 125, 14539.

[56] N. A. Patankar, Langmuir 2004, 20, 8209.

[57] J. T. Hirvi, T. A. Pakkanen, J. Phys. Chem. B 2007, 111, 3336

[58] C. T. Hsieh, J. M. Chen, R. R. Kuo, T. S. Lin, C. F. Wu, Appl. Surf. Sci. 2005, 240, 318 .

[59] M. Manca, B. Cortese, I. Viola, A. S. Arico, R. Cingolani, G. Gigli, Langmuir 2008, 24, 1833.

[60] D. K. Owens, R. C. Wendt, J. Appl. Polym. Sci. 1969, 13, 1741.

[61] MicroChem Corp. Website, http://www.microchem.com/products. (last accessed December 2008). 